\title{
Applying spelling error correction techniques for improving semantic role labelling
}

\author{
Erik Tjong Kim Sang \\ Informatics Institute \\ University of Amsterdam, Kruislaan 403 \\ NL-1098 SJ Amsterdam, The Netherlands \\ eriktescience.uva.nl
}

\author{
Sander Canisius, Antal van den Bosch, Toine Bogers \\ ILK / Computational Linguistics and AI \\ Tilburg University, P.O. Box 90153, \\ NL-5000 LE Tilburg, The Netherlands \\ \{S.V.M.Canisius, Antal.vdnBosch, \\ A.M.Bogers\}@uvt.nl
}

the number of instances at a reasonable number, we have only built instances for verb-phrase pairs when the phrase parent is an ancestor of the verb $(400,128$ training instances). A reasonable number of arguments are individual words; these do not match with phrase boundaries. In order to be able to label these, we have also generated instances for all pairs of verbs and individual words using the same constraint (another 542,217 instances). The parent node constraint makes certain that embedded arguments, which do not occur in these data sets, cannot be predicted by our approach.

Instances which are associated with verbargument pairs receive the label of the argument as class while others in principle receive a NULL class. In an estimated $10 \%$ of the cases, the phrase boundaries assigned by the parser are different from those in the argument annotation. In case of a mismatch, we have always used the argument label of the first word of a phrase as the class of the corresponding instance. By doing this we attempt to keep the positional information of the lost argument in the training data. Both the parser phrase boundary errors as well as the parent node constraint restrict the number of phrases we can identify. The maximum recall score attainable with our phrases is $84.64 \%$ for the development data set.

We have experimentally evaluated 30 features based on the previous work in semantic role labelling (Gildea and Jurafsky, 2002; Pradhan et al., 2004; Xue and Palmer, 2004):

- Lexical features (5): predicate (verb), first phrase word, last phrase word and words immediately before and after the phrase.

- Syntactic features (14): part-of-speech tags (POS) of: first phrase word, last phrase word, 
word immediately before phrase and word immediately after phrase; syntactic paths from word to verb: all paths, only paths for words before verb and only paths for words after verb; phrase label, label of phrase parent, subcategorisation of verb parent, predicate frame from PropBank, voice, head preposition for prepositional phrases and same parents flag.

- Semantic features (2): named entity tag for first phrase word and last phrase word.

- Positional features (3): position of the phrase with respect to the verb: left/right, distance in words and distance in parent nodes.

- Combination features (6): predicate + phrase label, predicate + first phrase word, predicate + last phrase word, predicate + first phrase POS, predicate + last phrase POS and voice + left/right.

The output of two parsers was available. We have briefly experimented with the Collins parses including the available punctuation corrections but found that our approach reached a better performance with the Charniak parses. We report only on the results obtained with the Charniak parses.

\section{Approach}

This section gives a brief overview of the three main components of our approach: machine learning, automatic feature selection and post-processing by a novel procedure designed to clean up the classifier output by correcting obvious misclassifications.

\subsection{Machine learning}

The core machine learning technique employed, is memory-based learning, a supervised inductive algorithm for learning classification tasks based on the $k$-nn algorithm. We use the TiMBL system (Daelemans et al., 2003), version 5.0.0, patch-2 with uniform feature weighting and random tiebreaking (options: -w 0 -R 911). We have also evaluated two alternative learning techniques. First, Maximum Entropy Models, for which we employed Zhang Le's Maximum Entropy Toolkit, version 20041229 with default parameters. Second, Support Vector Machines for which we used Taku Kudo's YamCha (Kudo and Matsumoto, 2003), with one-versus-all voting and option $-\mathrm{V}$ which enabled us to ignore predicted classes with negative distances.

\subsection{Feature selection}

In previous research, we have found that memorybased learning is rather sensitive to the chosen features. In particular, irrelevant or redundant features may lead to reduced performance. In order to minimise the effects of this sensitivity, we have employed bi-directional hill-climbing (Caruana and Freitag, 1994) for finding the features that were most suited for this task. This process starts with an empty feature set, examines the effect of adding or removing one feature and then starts a new iteration with the set associated with the best performance.

\subsection{Automatic post-processing}

Certain misclassifications by the semantic rolelabelling system described so far lead to unlikely and impossible relation assignments, such as assigning two indirect objects to a verb where only one is possible. Our proposed classifier has no mechanism to detect these errors. One solution is to devise a postprocessing step that transforms the resulting role assignments until they meet certain basic constraints, such as the rule that each verb may have only single instances of the different roles assigned in one sentence (Van den Bosch et al., 2004).

We propose an alternative automatically-trained post-processing method which corrects unlikely role assignments either by deleting them or by replacing them with a more likely one. We do not do this by knowledge-based constraint satisfaction, but rather by adopting a method for error correction based on Levenshtein distance (Levenshtein, 1965), or edit distance, as used commonly in spelling error correction. Levenshtein distance is a dynamically computed distance between two strings, accounting for the number of deletions, insertions, and substitutions needed to transform the one string into the other. Levenshtein-based error correction typically matches a new, possibly incorrect, string to a trusted lexicon of assumedly correct strings, finds the lexicon string with the smallest Levenshtein distance to the new string, and replaces the new string with the lexicon string as its likely correction. We implemented a roughly similar procedure. First, we generated a lexicon of semantic role labelling patterns of A0-A5 arguments of verbs on the basis of the entire training corpus and the PropBank verb frames. This 
lexicon contains entries such as abandon A0 V A1, and categorize A1 V A2 - a total of 43,033 variablelength role labelling patterns.

Next, given a new test sentence, we consider all of its verbs and their respective predicted role labellings, and compare each with the lexicon, searching the role labelling pattern with the same verb at the smallest Levenshtein distance (in case of an unknown verb we search in the entire lexicon). For example, in a test sentence the pattern emphasize $\mathrm{A} 0$ $\mathrm{VA} A \mathrm{~A} 0$ is predicted. One closest lexicon item is found at Levenshtein distance 1, namely emphasize $\mathrm{A} 0 \mathrm{VA} \mathrm{A}$, representing a deletion of the final $\mathrm{A} 0$. We then use the nearest-neighbour pattern in the lexicon to correct the likely error, and apply all deletions and substitutions needed to correct the current pattern according to the nearest-neighbour pattern from the trusted lexicon. We do not apply insertions, since the post-processor module does not have the information to decide which constituent or word would receive the inserted label. In case of multiple possible deletions (e.g. in deleting one out of two A1s in emphasize A0 V A1 A1), we always delete the argument furthest from the verb.

\section{Results}

In order to perform the optimisation of the semantic role labelling process in a reasonable amount of time, we have divided it in four separate tasks: pruning the data for individual words and the data for phrases, and labelling of these two data sets. Pruning amounts to deciding which instances correspond with verb-argument pairs and which do not. This resulted in a considerable reduction of the two data sets: $47 \%$ for the phrase data and $80 \%$ for the word data. The remaining instances are assumed to define verb-argument pairs and the labelling tasks assign labels to them. We have performed a separate feature selection process in combination with the memory-based learner for each of the four tasks. First we selected the best feature set based on task accuracy. As soon as a working module for each of the tasks was available, we performed an extra feature selection process for each of the modules, optimising overall system $\mathrm{F}_{\beta=1}$ while keeping the other three modules fixed.

The effect of the features on the overall perfor-

\begin{tabular}{|l|c|c|c|c|}
\cline { 2 - 5 } \multicolumn{1}{c|}{} & \multicolumn{2}{c|}{ Words } & \multicolumn{2}{c|}{ Phrases } \\
\hline Features & prune & label & prune & label \\
\hline predicate & -0.04 & $\mathbf{+ 0 . 0 5}$ & -0.25 & -0.52 \\
first word & $\mathbf{+ 0 . 3 8}$ & $\mathbf{+ 0 . 1 6}$ & -0.17 & $\mathbf{+ 1 . 1 4}$ \\
last word & - & - & -0.01 & $\mathbf{+ 1 . 1 2}$ \\
previous word & -0.06 & $\mathbf{+ 0 . 0 2}$ & -0.05 & $\mathbf{+ 0 . 7 4}$ \\
next word & -0.04 & -0.08 & $\mathbf{+ 0 . 4 4}$ & -0.16 \\
part-of-speech first word & -0.01 & -0.02 & -0.07 & -0.11 \\
part-of-speech last word & - & - & -0.14 & -0.45 \\
previous part-of-speech & -0.12 & -0.06 & $\mathbf{+ 0 . 2 2}$ & -1.14 \\
next part-of-speech & -0.08 & -0.12 & -0.01 & -0.21 \\
all paths & $\mathbf{+ 0 . 4 2}$ & $\mathbf{+ 0 . 1 0}$ & $\mathbf{+ 0 . 8 4}$ & $\mathbf{+ 0 . 7 5}$ \\
path before verb & +0.00 & -0.02 & +0.00 & $\mathbf{+ 0 . 2 7}$ \\
path after verb & -0.01 & -0.01 & -0.01 & -0.06 \\
phrase label & -0.01 & -0.02 & $\mathbf{+ 0 . 1 3}$ & -0.02 \\
parent label & $\mathbf{+ 0 . 0 3}$ & -0.02 & -0.03 & +0.00 \\
voice & +0.02 & -0.04 & -0.04 & $\mathbf{+ 1 . 8 5}$ \\
subcategorisation & -0.01 & +0.00 & -0.02 & +0.03 \\
PropBank frame & -0.12 & -0.03 & -0.16 & $\mathbf{+ 1 . 0 4}$ \\
PP head & +0.00 & +0.00 & -0.06 & $\mathbf{+ 0 . 0 8}$ \\
same parents & -0.02 & -0.01 & $\mathbf{+ 0 . 0 3}$ & -0.05 \\
named entity first word & +0.00 & +0.00 & $\mathbf{+ 0 . 0 5}$ & -0.11 \\
named entity last word & - & - & -0.04 & -0.12 \\
absolute position & $\mathbf{+ 0 . 0 0}$ & +0.00 & +0.00 & -0.02 \\
distance in words & $\mathbf{+ 0 . 3 4}$ & $\mathbf{+ 0 . 0 4}$ & $\mathbf{+ 0 . 1 6}$ & -0.96 \\
distance in parents & -0.02 & -0.02 & $\mathbf{+ 0 . 0 6}$ & -0.04 \\
predicate + label & -0.05 & -0.07 & -0.22 & -0.47 \\
predicate + first word & -0.05 & +0.00 & $\mathbf{+ 0 . 1 3}$ & $\mathbf{+ 0 . 9 7}$ \\
predicate + last word & - & - & -0.03 & $\mathbf{+ 0 . 0 8}$ \\
predicate + first POS & -0.05 & -0.06 & -0.20 & -0.50 \\
predicate + last POS & - & - & -0.13 & -0.40 \\
voice + position & +0.02 & -0.04 & -0.05 & -0.04 \\
\hline
\end{tabular}

Table 1: Effect of adding a feature to the best feature sets when memory-based learning is applied to the development set (overall $\mathrm{F}_{\beta=1}$ ). The process consisted of four tasks: pruning data sets for individual words and phrases, and labelling these two data sets. Selected features are shown in bold. Unfortunately, we have not been able to use all promising features.

mance can be found in Table 1. One feature (syntactic path) was selected in all four tasks but in general different features were required for optimal performance in the four tasks. Changing the feature set had the largest effect when labelling the phrase data. We have applied the two other learners, Maximum Entropy Models and Support Vector Machines to the two labelling tasks, while using the same features as the memory-based learner. The performance of the three systems on the development data can be found in Table 3. Since the systems performed differently we have also evaluated the performance of a combined system which always chose the majority class assigned to an instance and the class of the strongest system (SVM) in case of a three-way tie. The combined system performed slightly better than the best 


\begin{tabular}{|l|r|r|r|}
\cline { 2 - 4 } \multicolumn{1}{c|}{} & Precision & Recall & $\mathrm{F}_{\beta=1}$ \\
\hline Development & $76.79 \%$ & $70.01 \%$ & 73.24 \\
Test WSJ & $79.03 \%$ & $72.03 \%$ & 75.37 \\
Test Brown & $70.45 \%$ & $60.13 \%$ & 64.88 \\
Test WSJ+Brown & $77.94 \%$ & $70.44 \%$ & 74.00 \\
\hline
\end{tabular}

\begin{tabular}{|l|r|r|r|}
\cline { 2 - 4 } \multicolumn{1}{c|}{ Test WSJ } & Precision & Recall & $\mathrm{F}_{\beta=1}=1$ \\
\hline Overall & $79.03 \%$ & $72.03 \%$ & 75.37 \\
\hline A0 & $85.65 \%$ & $81.73 \%$ & 83.64 \\
A2 & $76.97 \%$ & $71.89 \%$ & 74.34 \\
A3 & $71.07 \%$ & $58.20 \%$ & 63.99 \\
A4 & $69.29 \%$ & $50.87 \%$ & 58.67 \\
A5 & $75.56 \%$ & $66.67 \%$ & 70.83 \\
AM-ADV & $100.00 \%$ & $40.00 \%$ & 57.14 \\
AM-CAU & $64.36 \%$ & $51.38 \%$ & 57.14 \\
AM-DIR & $75.56 \%$ & $46.58 \%$ & 57.63 \\
AM-DIS & $81.98 \%$ & $28.24 \%$ & 35.82 \\
AM-EXT & $87.50 \%$ & $79.06 \%$ & 80.45 \\
AM-LOC & $62.50 \%$ & $43.75 \%$ & 58.33 \\
AM-MNR & $64.52 \%$ & $52.33 \%$ & 57.15 \\
AM-MOD & $96.76 \%$ & $97.64 \%$ & 97.20 \\
AM-NEG & $97.38 \%$ & $96.96 \%$ & 97.17 \\
AM-PNC & $45.98 \%$ & $34.78 \%$ & 39.60 \\
AM-PRD & $50.00 \%$ & $20.00 \%$ & 28.57 \\
AM-REC & $0.00 \%$ & $0.00 \%$ & 0.00 \\
AM-TMP & $80.52 \%$ & $70.75 \%$ & 75.32 \\
R-A0 & $81.47 \%$ & $84.38 \%$ & 82.89 \\
R-A1 & $74.00 \%$ & $71.15 \%$ & 72.55 \\
R-A2 & $60.00 \%$ & $37.50 \%$ & 46.15 \\
R-A3 & $0.00 \%$ & $0.00 \%$ & 0.00 \\
R-A4 & $0.00 \%$ & $0.00 \%$ & 0.00 \\
R-AM-ADV & $0.00 \%$ & $0.00 \%$ & 0.00 \\
R-AM-CAU & $100.00 \%$ & $25.00 \%$ & 40.00 \\
R-AM-EXT & $100.00 \%$ & $100.00 \%$ & 100.00 \\
R-AM-LOC & $86.67 \%$ & $61.90 \%$ & 72.22 \\
R-AM-MNR & $33.33 \%$ & $33.33 \%$ & 33.33 \\
R-AM-TMP & $64.41 \%$ & $73.08 \%$ & 68.47 \\
\hline \hline V & $97.36 \%$ & $97.36 \%$ & 97.36 \\
\hline
\end{tabular}

Table 2: Overall results (top) and detailed results on the WSJ test (bottom).

individual system.

\section{Conclusion}

We have presented a machine learning approach to semantic role labelling based on full parses. We have split the process in four separate tasks: pruning the data bases of word-based and phrase-based examples down to only the positive verb-argument cases, and labelling the two positively classified data sets. A novel automatic post-processing procedure based on spelling correction, comparing to a trusted lexicon of verb-argument patterns from the training material, was able to achieve a performance increase by correcting unlikely role assignments.

\begin{tabular}{|c|c|c|c|}
\hline Learning algorithm & Precision & Recall & $\mathrm{F}_{\beta=1}$ \\
\hline \multicolumn{4}{|l|}{ without post-processing: } \\
\hline Maximum Entropy Models & $70.78 \%$ & $70.03 \%$ & 70.40 \\
\hline Memory-Based Learning & $70.70 \%$ & $69.85 \%$ & 70.27 \\
\hline Support Vector Machines & $75.07 \%$ & $69.15 \%$ & 71.98 \\
\hline \multicolumn{4}{|l|}{ including post-processing: } \\
\hline Maximum Entropy Models & $74.06 \%$ & $69.84 \%$ & 71.89 \\
\hline Memory-Based Learning & $73.84 \%$ & $69.88 \%$ & 71.80 \\
\hline Support Vector Machines & $77.75 \%$ & $69.11 \%$ & 73.17 \\
\hline Combination & $76.79 \%$ & $70.01 \%$ & 73.24 \\
\hline
\end{tabular}

Table 3: Effect of the choice of machine learning algorithm, the application of Levenshtein-distancebased post-processing and the use of system combination on the performance obtained for the development data set.

\section{Acknowledgements}

This research was funded by NWO, the Netherlands Organisation for Scientific Research, and by SenterNovem IOP-MMI.

\section{References}

X. Carreras and L. Màrquez. 2005. Introduction to the CoNLL2005 Shared Task: Semantic Role Labeling. In Proceedings of CoNLL-2005. Ann Arbor, MI, USA.

R. Caruana and D. Freitag. 1994. Greedy attribute selection. In Proceedings of the Eleventh International Conference on Machine Learning, pages 28-36, New Brunswick, NJ, USA. Morgan Kaufman.

W. Daelemans, J. Zavrel, K. van der Sloot, and A. van den Bosch. 2003. TiMBL: Tilburg memory based learner, version 5.0, reference guide. ILK Technical Report 03-10, Tilburg University.

D. Gildea and D. Jurafsky. 2002. Automatic labeling of semantic roles. Computational Linguistics, 28(3):245-288.

T. Kudo and Y. Matsumoto. 2003. Fast methods for kernelbased text analysis. In Proceedings of ACL-2003. Sapporo, Japan.

V. Levenshtein. 1965. Binary codes capable of correcting deletions, insertions and reversals. Doklady Akademii Nauk SSSR, 163(4):845-848.

S. Pradhan, W. Ward, K. Hacioglu, J. Martin, and D. Jurafsky. 2004. Shallow semantic parsing using support vector machines. In Proceedings of the HLT/NAACL 2004. Boston, MA.

A. van den Bosch, S. Canisius, W. Daelemans, I Hendrickx, and E. Tjong Kim Sang. 2004. Memory-based semantic role labeling: Optimizing features, algorithm, and output. In Proceedings of the CoNLL-2004, Boston, MA, USA.

N. Xue and M. Palmer. 2004. Calibrating features for semantic role labeling. In Proceedings of EMNLP-2004. Barcelona, Spain. 